\title{
Extremum Seeking Tuning for Reel Tension Control in Haptic Application
}

\author{
Martin J.-D. Otis Thien-Ly Nguyen-Dang Denis Laurendeau* \\ Clément Gosselin ** \\ * Department of Electrical Engineering, Université Laval, Québec City, QC, \\ Canada (e-mail: laurend@gel.ulaval.ca). \\ ** Department of Mechanical Engineering, Université Laval, Québec City, \\ QC, Canada (e-mail: gosselin@gmc.ulaval.ca).
}

\begin{abstract}
Optimal tension control in parallel mechanisms for balancing wrench at the end-effector introduces a complex model of the overall system. Moreover, actuator saturations such as motor current and angular velocity limits impose that a non-linear system be controlled. Such a system is usually controlled sub-optimally due to the complexity of its model. In haptic applications where transparency and stability are both critical parameters, optimal performance should be achieved. This paper introduces an enhanced FPID auto-tuning based on Extremum Seeking-Tuning (ES-Tuning) which uses a different cost function to find the local optimal solution for haptic rendering and a correction on noise measurement for a high order complex system. The solution of this optimization gives a direct measure of the reel dynamic transparency evaluated by the proposed cost function.
\end{abstract}

Keywords: PID Tuning, Optimal cable tensions control, Reel transparency, Haptic.

\section{INTRODUCTION}

$\mathbf{T}$ Ransparency is associated with the absence of friction, inertia and gravity force or any other parasitic wrench that could be sensed during the motion of a haptic device (Vlachos and Papadopoulos [2006]). The dynamic range of achievable impedance (Z-width) is also a measure of the transparency performance (Colgate and Brown [1994]).

When modeling a redundant cable-driven parallel mechanism system in order to properly employ an optimal tension distribution algorithm as in Fang et al. [2004] or quadratic programming as in Oh and Agrawal [2005], the cables are considered as being simple line segments unaffected by gravity. However, for the cable real behaviour described in Ali [1986] and Baicu et al. [1996], the mechanical elastic deformation of the reel and the overall structure as well as the cable tension measure produce a high order system for which optimal control is difficult to achieve with standard tuning methods.

In this paper, a discrete version of ES-Tuning according to Killingsworth and Krstic [2005] is used for optimizing the transparency of a reel response controlled in cable tension by minimizing a cost function. The design of the cost function defines the performance of the reel estimated by a measure on the dynamic response and on the tension error. The reel design uses low cost motor and acquisition system generating noise on the measurements. The noise signature fed to the optimization procedure reduces the effectiveness of the algorithm. Thus, the final optimization of the FPID parameters of the reel controller performed using a slightly modified version of the known adaptive ES-Tuning algorithm for increasing robustness. Other non-linear models based tuning method like iterative feedback tuning (IFT) could be implemented as described in Lequin

${ }^{1}$ This work was supported by the Natural Sciences and Engineering Research Council of Canada (NSERC). et al. [2003]. Both methods give similar results according to Killingsworth and Krstic [2006] but the optimization procedure seems to be more time effective with ES-Tuning as it is mentioned in Killingsworth et al. [2007].

The second section of this paper presents a second order model of the reel transfer function for evaluating the parameters of a first FPID (filter+PID). The third section presents the control architecture for cable tension control. The fourth section introduces an enhanced FPID auto-tuning algorithm based on Extremum Seeking-Tuning which uses a cost function designed for haptic applications that finds a local optimal solution and a correction on noise measurement.

\subsection{Acquisition System}

The chosen force controller architecture consists of a slightly modified FPID core that includes feed-forward compensation for friction, inertia and cable gravity. The output of the FPID core, which corresponds to a given torque value, is then fed into a current controller that is linearized in software using polynomial least-squares regression (as it gives a very efficient method for evaluating the linearizing function without consuming as much memory and processor time as a trilinear interpolated lookup table). The current controller operates in specialized hardware separate from the main control computer, and its response speed and range implies that it is acceptable to ignore the effect of inductance inside the reel DC motor an assumption that greatly simplifies transfer function calculations. Finally, the data from the strain gauge is acquired with 4-20mA amplifier, and a curve determined from quadratic least-squares regression is used to transform the raw sensor values into force (and thus torque) values. 


\section{REEL TRANSFER FUNCTION}

Since the main hardware interfacing the QNX computer and the reels uses current control, the transfer function of the motor torque with respect to a DAC control signal can be considered (after linearization) as being a simple constant multiplicator, and thus the non-instantaneous response measured at the strain gauge for a Heaviside-like setpoint curve is mainly due to the elastic properties of the strain gauge itself combined with the inertia of the reel drum and motor as well as the parasitic elastic contribution of the reel structure and the finite Young modulus of the affixed cable.

\subsection{Second Order Approximation}

As the strain gauge has negligible mass in comparison to the reel drum and the DC motor rotor component, it is possible to include the effects of its elasticity and the cable elasticity within an effective Hooke constant, which, combined with the inertia of all moving rotational parts (including the cable itself), allows for the calculation of an approximative under damped second-order transfer function $F(s)$ that can be used to model the system response at frequencies lower than the strain gauge resonant frequency (in this case, approximately $300 \mathrm{~Hz}$, and is thus comparable to the Nyquist frequency of the sampling subsystem):

$$
F(s)=T \frac{1}{s^{2}+\frac{b_{m}}{J_{m}} s+\frac{k_{m}}{J_{m}}}=T \frac{1}{s^{2}+2 \zeta \omega_{n} s+\omega_{n}^{2}},
$$

where $b_{m}$ is the effective viscous friction constant, $J_{m}$ is the total rotational inertia reflected to the motor axle, $k_{m}$ is the effective Hooke constant, $\zeta$ is the damping constant and $\omega_{n}$ is the natural frequency. This model was used as a starting point for determining initial FPID parameters before automatic fine tuning of the model during the calibration procedure.

\subsection{Experimental Estimation of the Transfert Function}

The experimental procedure used in the custom-made calibration software to determine the transfer function parameters mentioned above can be summarized as follows: the rotational inertia is calculated by measuring the maximum angular acceleration of the motor axle for a known precalibrated open-loop command. The viscous friction constant is then determined by waiting stabilization of motor speed at $80 \%$ of its maximal speed (in this case, it is limited in voltage by a clamp diode that determines the maximum allowed CEMF), then measuring the rate of decay of this speed for a null setpoint, and fitting an exponential curve using standard least-squares regression. Note that this assumes a first order angular velocity transfer function with respect to the FPID output command (which is a valid approximation because the reels are controlled in current). Finally, the effective Hooke constant is determined by either measuring the oscillating frequency $\omega_{\text {osc }}$ of the system for a Heaviside input setpoint, in which case:

$$
k_{m}=\frac{b_{m}^{2}}{4 J_{m}}+J_{m} \omega_{o s c}^{2},
$$

or one can also determine the Bode plot of the system so as to extrapolate the system resonant frequency $\omega_{R}$, in which case:

$$
k_{m}=\frac{b_{m}^{2}}{2 J_{m}}+J_{m} \omega_{R}^{2}
$$

\section{CONTROL ARCHITECTURE}

The chosen reel tension controller architecture is based on a slightly modified FPID closed-loop control scheme as described in Killingsworth and Krstic [2006] that includes all feed-forward compensation terms as well as an open-loop (and optional) setpoint filter, and whose core can be detailed as follows. Within the Laplace transform domain, let $G$ be the process to be controlled, $C_{y}$ a controller term, and $C_{r}$ its derivative-reduced counterpart:

$$
\begin{aligned}
& C_{y}(s)=K\left(1+\frac{1}{T_{i} s}+T_{d} s\right), \text { and } \\
& C_{r}(s)=K\left(1+\frac{1}{T_{i} s}\right)
\end{aligned}
$$

where the three PID parameters are $P=K, I=K / T_{i}$ and $D=K T_{d}$. The servo system is designed such that the closedloop transfer function $T(s)$ becomes:

$$
T(s)=\frac{G C_{r}(s)}{1+G C_{y}(s)} .
$$

The transfer function in (6) must be changed in practice so as to take into account the floating point calculation errors which increase monotonically with the magnitude of the numbers involved. In effect, Killingsworth suggests that controller $C_{r}$ be inserted between the input of a closed-loop controller and a given reference setpoint $r$, which is numerically equivalent to calculating the effects of an open-loop integrator $C_{r}$, and then by compensating its asymptotic ever-increasing behavior by subtracting another monotonically increasing integrator term $C_{y}$ to it. Although it is always possible to implement a circular accumulator buffer in order to compensate for this behavior, one can also notice that in the time domain, it is equivalent to calculate the derivative term separately from the integral and proportional term of the PID controller, all within a standard PID architecture.

\section{TUNING TENSION CONTROLLER}

The FPID controller parameters are optimized by minimizing a cost function locally that describes the accuracy of the controller output with respect to a given Heaviside-like setpoint. Two methods are investigated within the framework of a concrete robotic device, namely a modified version of the ES-Tuning algorithm which calculates the gradient of the cost function using a combination of high-pass and low-pass digital filters in order to extract the information from sine modulation of the FPID parameters $\left(F=\tau_{f}, P=K, I=K / T_{i}\right.$ and $D=$ $K T_{d}$ multiplicative constants), or rather using a standard algorithm that extrapolates this gradient using multidimensional least-squares regression of a set of neighboring points in the FPID parameter space. One expects the ES method to converge faster than the least-squares method, for the latter must poll a sufficient number of neighboring points to a given position in the FPID parameter space in order to calculate its gradient, but the discrepancy between the two methods was experimentally found to be quite small due to inherent measurement noise which favors robustness over convergence speed.

\subsection{Cost Functions and Transparency}

In a cable tension control application, overshoot should be avoided with a low damping response. A general rule in de- 
signing a meaningful cost function is usually to tune the settling time and the rise time of the controller response while minimizing overshoot. It is possible to combine both usual ISE and ITSE cost functions by using a sigmoid weighting function so that not only the transient portion is de-emphasized, but the subsequent settling phase basically corresponds to a constant weighing function, as in the ISE:

$$
J(t) \equiv \frac{I T S E+\frac{1}{t_{s}-t_{0}} \int_{t_{0}}^{t_{s}} \frac{1}{1+e^{\pi\left(t-t_{s}\right) /\left(2 t_{s}\right)}} \epsilon^{2} d t}{J(O(1))},
$$

where $J(t)$ is the cost function, $J(O(1))$ is the cost function evaluated for a first order system, $\epsilon$ is the error between the cable tension command (the reference $r$ ) and the strain gauge measure (the output $y$ ), $t_{s}$ is the settling time of the desired response and $\pi / 2$ adjusts the curve of the sigmoid. This reduces the need for fine tuning the constant $t_{s}$ to achieve the desired response. Instead, it is chosen as a value that is extrapolated directly from the natural oscillation frequency of the second-order model using the simple relation $t_{s} \leq 2 \pi / \omega_{n}$. The conditions $J<J_{\max }$ must be verified ensuring that the algorithm does not enter in a unstable region. $J_{\max }$ is determined experimentally.

$J$ gives a measure on the haptic transparency and the performance of the reel with a consideration on the dynamic (settling time $t_{s}$ ) and on the cable tension error $\epsilon$.

\subsection{Implementation of ES-Tuning}

The modifications to the ES tuning algorithm stem from the necessity of adapting the procedure of Killingsworth and Krstic [2006] to a concrete cable-actuated robotic system in which the imperfect repeatability of the reel output for a given setpoint translates into a cost function measurement noise that must be taken into account in order to ensure convergence to the desired local minimizer. The setpoint filter time constant $\tau_{f}$ is added as an additional tunable parameter so that the smoothness of the FPID response can be adjusted depending on the cost function employed. Moreover, to restrict the parameters to positive values and to allow their tunability for a wide range of starting parameter space coordinates, the natural logarithm of each parameter is fed in the tuning algorithm, as this eliminates the pathological behavior for very small parameter values where the discretization step of the numerical gradient integrator becomes significant. This procedure somewhat relaxes the constraints on the choice of the three ES-Tuning constants, namely the modulation index $\alpha_{i}$, the modulation frequency $\omega_{i}$ and the gradient integrator constant $\gamma_{i}$ for each FPID parameter $(i$ being a label for each parameter $\tau_{f}, P, I$ and $\left.D\right)$. The discrete-time ES algorithm proposed in this paper is resumed in (8) to (12):

$$
\begin{aligned}
\theta_{[k]} & \equiv\left[\left(\log _{e} P\right)\left(\log _{e}(1 / I)\right)\left(\log _{e} D\right)\left(\log _{e} \tau_{f}\right)\right], \\
\chi_{[k]} & =-h \chi_{[k-1]}+\eta\left(J_{[k]}-J_{[k-1]}\right), \\
\beta_{i[k]} & =\chi_{[k]} \alpha_{i} \cos \left(k \omega_{i}\right), \\
\hat{\theta}_{i[k+1]} & =\hat{\theta}_{i[k]}-\gamma_{i} \beta_{i[k]} / J(\theta)_{[k]} \text { and } \\
\theta_{i[k+1]} & =\hat{\theta}_{i[k+1]}+\alpha_{i} \cos \left((k+1) \omega_{i}\right)
\end{aligned}
$$

where $\theta_{i[k]}$ is the parameter that needs to be found, $\chi_{[k]}$ is the filtered signal of $J(\theta)_{[k]}, \beta_{i[k]}$ is the demodulated signal and $\hat{\theta}_{i[k]}$ is the value of the estimate at iteration $k$. The effect of adding multiplication factors $\eta$ and $1 / J(\theta)_{[k]}$ on the filters will be explained in the next sections. Also, in section 4.5, a low pass filter is inserted to these equations for decreasing the noise on $\chi_{[k]}$.

\subsection{ES-Tuning Parameters}

To determine the initial PID and $\tau_{f}$ parameters to be used at the beginning of the tuning procedure, a second-order transfer function can be used in an empirical polynomial regressionbased model as defined in Shen and Chiang [2004] in order to approximate the optimal parameters. As for the ES-Tuning constants $\alpha_{i}, \omega_{i}, \gamma_{i}$ and the filter settings $\left|T_{h}\right|$ and $\left|T_{l}\right|$, they must be determined so as not only obtain the best possible settling time, but also minimize the now-present and unavoidable noise signature.

The high-pass filter must indeed be set so that the cutoff frequency be the highest possible, in order to speed up the filter impulse response in the time domain. This high-pass filter has the following structure:

$$
\begin{aligned}
T_{h} & =\eta \frac{z-1}{z+h}, \text { with } \\
\eta & =\sqrt{\frac{(h-1)^{2}}{4}}
\end{aligned}
$$

$\eta$ is the normalization factor, and

$$
h=\left\{\begin{array}{cc}
\frac{\sin \omega_{c}-1}{\cos \omega_{c}} & \text { if } \omega_{c} \neq \pi / 2 \\
0 & \text { otherwise }
\end{array}\right.
$$

defines the cut-off frequency. This filter can be defined in the time domain with the following recursive function:

$$
y[n]-h y[n-1]=\eta(x[n]-x[n-1])
$$

and its settling time can be characterized by analysing its response to a Heaviside step $x[n]=H[n]$, in which case $x[n]-$ $x[n-1]=0 \forall n>0$, and $y[0]=\eta$. This means that:

$$
y[n]=h y[n-1]=(\operatorname{sgn} h)^{n}|h|^{n} \eta
$$

and thus that the settling time (defined as $N_{h}$ for $\left|y\left[N_{h}\right]\right|=$ $\left.e^{-1} \eta\right)$ is:

$$
N_{h}=\log _{h}|h|^{N_{h}}=-\lim _{l \rightarrow|h|} \frac{1}{\ln (l)} .
$$

The constraint on $h$ is that $\omega_{c} \leq \min \left(\omega_{i}\right)$ so that the modulated components of $J$ are not filtered out. It thus seems that choosing the lowest frequency $\omega_{i}$ to be higher than $\pi / 2$ would be the best course of action (so that $N_{h}$ is minimized for $h=0$ ), but it will be soon clear that it is not necessarily the case. When the demodulation process is completed and the amplitude of the modulated signal at $\omega_{i}$ is shifted to the baseband (DC component), it is imperative to cut not only the other frequency components $\omega_{j \neq i}$ using a low-pass filter, but also all harmonics that stem from the nonlinearity of $J$ with respect to its parameters. Actually, $J$ can be expressed as a Taylor series, and thus all sinusoidal components of the modulated signals will be crossmultiplied together to a certain extent when passing through $J$, unless the amplitudes $\alpha_{i}$ are kept the smallest possible. Note that the closer $\omega_{i}$ are to the Nyquist frequency, the closer they will be to each other, which means that the low-pass filter will need to be of higher quality in order to cut the undesirable baseband harmonics (after demodulation). The issue is that $\alpha_{i}$ must be lower-bounded to ensure that the noise level does not 
drown the desired modulated signals. Hence, it is impossible to avoid using a low-pass filter.

\subsection{Selecting Parameters for Tuning}

According to the original ES-Tuning design, the low-pass filter is simply the integrator of the extremum-seeking algorithm, in which case the frequency response is:

$$
\left|T_{l}\right|=\frac{\gamma}{\sqrt{2(1-\cos \omega)}},
$$

with a null response at exactly the Nyquist frequency. If the integrator is allowed to sum the DC component for a long enough time, then the frequency components not in the baseband will be effectively filtered out. However the time required for the integration of baseband signal from $\omega_{i}$ must be much greater than the period of all components in the baseband-shifted spectrum. Moreover, any short-term oscillation of the integrator caused by those undesirable harmonics can potentially interfere with the modulation process of cost function $J$, unless care is taken to either ensure that the baseband harmonics do not match any value $\omega_{i}$, or by greatly reducing the integration terms $\gamma_{i}$ so that the incremental change of $J$ is insignificant next to the modulation amplitude of $J$. More precisely, if it is determined that a parameter modulation amplitude $\alpha_{i}$ causes a cost function oscillation of amplitude $\beta_{i}\left(\alpha_{i}\right)$, then the ideal baseband DC component after demodulation and perfect lowpass filtering would have an amplitude $\beta_{i}\left(\alpha_{i}\right)$. Thus $\gamma_{i}<$ $\kappa_{i} \alpha_{i} / \max \left(\beta_{i}\left(\alpha_{i}\right)\right)$, where $0<\kappa_{i} \ll 1$ should be satisfied, thus limiting the speed at which $J$ can converge. As a valid rule of thumb, $\kappa_{i}$ can be tuned so that the parameter variation after $2 \pi / \min \left(\omega_{i}\right)$ samples is less than $\alpha_{i}$, i.e. $\kappa_{i}<\min \left(\omega_{i}\right) / 2 \pi$. It is possible to choose the frequency components $\omega_{i}$ so that in the case where the nonlinearity of $J$ is limited to a quadratic term, the resulting frequency terms $\left(\omega_{i}, 2 \omega_{i}, \omega_{i}+\omega_{j}, \omega_{i}-\omega_{j}\right)$ do not overlap the fundamental frequencies $\omega_{i}$ for all $i$. This is why the relation $\omega_{i}=\pi a^{i}$, where $0<a<1$, is used.

Suppose that a design requires a high-pass filter with the fastest possible settling time. Then, in order to ensure that the lowest frequency for a four parameters FPID system $\left(\omega_{4}\right)$ is higher than $\pi / 2, a$ is chosen so that $a^{1 / 4}>0.5$, i.e. $a>0.841$. An example of an acceptable value would then be $a=0.88$, for which the maximum distance between harmonics and the desired frequencies $\omega_{i}$, considering the effect of aliasing, is numerically verified to be at minimum $0.04 \pi$.

As for the noise considerations, it is imperative to ensure that the amplitudes $\beta_{i}$ are sufficient so that they be distinguishable from what is assumed to be Gaussian noise over the frequency range $0 \leq \omega \leq \pi$. Therefore, constant minimal SNR ratios $\varsigma_{i}$ between components $\beta_{i}$ and the actual noise of the cost function $J$ considered as acceptable are defined, on which all other parameters will be based. Knowing that noise is found in the demodulated signal and that it is in the short term transmitted through the integrators, thereby directly affecting the value of $J$ for the next iterations, the condition $\varsigma_{i}>\varsigma$ for the worst case $\kappa_{i}=1$, where $\varsigma \geq 1$ is a user-determined constant describing the overall quality of the cost function, is imposed for all $\beta_{i}$. Indeed, this at least assures that for the case $\varsigma=1$ there is no random walk in the parameter space, because the baseband DC signal corresponding to each $\omega_{i}$ to be integrated will always at least have the correct sign. In the case where $\kappa_{i}<1$, only the part of the noise spectrum below the critical frequency $\pi \kappa_{i}$ must be taken into account, in which case the noise power spectrum is scaled by $\kappa_{i}$, and thus $\varsigma_{i}>\varsigma \kappa_{i}$.

Note that a more precise assessment of $\varsigma_{i}$ would also consider the reduction of the total noise power due to the high-pass filtering, but this actually results in less stringent conditions, and thus does not adversely affects the result. In other terms, the effect of the high-pass filter can be considered as a safety margin for the algorithm.

Keeping all these constraints in mind, and that the uncertainties of the optimal FPID parameter values in logarithmic space found by ES-Tuning are ultimately proportional to $\alpha_{i}$, these values are chosen to be smaller than a relative precision value $\varepsilon$, which in the base-e logarithm space is in this case fixed to 0.07 (i.e. a scaling factor of 0.93 or 1.07 approximately with respect to the magnitude of the parameters). Such a small value not only results in a good precision for the optimal parameters, but also ensures that the local variation of parameters are to a first approximation bilinear (and not logarithmic). However, it is likely to be insufficient to cope with the cost function noise, which means that additional measures must be taken to reduce its influence.

\subsection{Noise Reduction}

The most straightforward way to act on the SNR of the cost function is simply to sample it $N$ times for an amplitude noise level attenuation of $\sqrt{N}$, at the cost of scaling up the settling time of the algorithm by a factor $N$. The second method is to implement an additional low-pass filter between the demodulator and the integrator, so as to filter out the highfrequency noise while reducing the impact of harmonics in the DC baseband integration step (a very desirable feature indeed). Consider the IIR low-pass filter of the form:

$$
T_{l}=\frac{1+z^{-1}}{\left(1+\frac{\omega_{c}}{\pi}\right)+z^{-1}\left(1-\frac{\omega_{c}}{\pi}\right)} .
$$

The time domain properties of this filter can be determined easily by observing that this is the Bilinear transform of the equivalent Laplace transform of a simple first-order filter with cutoff frequency corresponding to its digital counterpart $\omega_{c}$. Note that prewarping can be ignored because $\omega_{c} \ll \pi$, which is required in order for the filter to become an effective noise and spurious harmonics suppressor. In other terms, the settling time can be described by $N_{l}=2 \pi / \omega_{c}$.

As for the noise attenuation properties, one observes that the amplitude noise level attenuation is approximatively proportional to $\sqrt{2 \pi / \omega_{c}}$, and is thus also proportional to the settling time of the filter. Therefore, the additional low-pass filtering is the favored method, because in contrary to the multiple sampling method, it allows to lower the high-pass filter settling time by setting $N_{l}=N_{h}$, therefore resulting in the use of lower frequency values of $\omega_{i}$, which in turn improves the discrimination capacity of the low-pass filter.

\section{ENHANCED ES-TUNING ALGORITHM}

To summarize the new additions to the ES-Tuning algorithm, here is a short description of a possible configuration algorithm:

- the four initial parameters $\theta_{i[k=0]}$ are set according to Shen and Chiang [2004]; 
- the values of $\alpha_{i}$ are set to the chosen value $\varepsilon \approx 0.07$;

- the SEM (i.e. noise) and the corresponding standard deviation of the cost function are evaluated by calculating the cost function for a Heaviside setpoint at the initial FPID parameters repeatedly after the output of the controller settles to a stable average value;

- initiate a very high Q high-pass filter and very high Q lowpass filters, then modulate the parameters individually with their respective amplitudes $\alpha_{i}$. Proceed with demodulation, but do not integrate the filtered DC baseband component with amplitude $\beta_{i}$. After time $N_{h}$, note the value of this component for each $\omega_{i}$.

- calculate $\varsigma / \min \left(\varsigma_{i r}\right)$, where $\varsigma_{i r}$ are the raw SNR value of the cost function $J$ for every parameter. Knowing that $\varsigma_{i}>\varsigma \kappa_{i}$, and that $\kappa_{i}^{-1}=N_{h}=N_{l}$ is possible, one concludes that $\kappa_{i}^{-1}=N_{h}=N_{l}=\sqrt{\varsigma / \min \left(\varsigma_{i r}\right)}$;

- the angular frequency constant $\omega_{i}$ for each parameter is set according to the rule in Killingsworth and Krstic [2006] where $\omega_{i}=\pi a^{i}$, for $0<a<1$, so as to minimize nonlinear mathematical coupling between the different spectrum components. Determine the minimum value of $a$ which maximizes the distance between baseband and aliased harmonics of the cost function $J$ using a pre-calculated lookup table (for 1 st and second order harmonics only);

- initialize the high-pass filter, the low-pass filters and the integration parameter according to the chosen value of $\kappa_{i}$. Allow the high-pass filter buffer to settle to the initial value of $J$ by deactivating the integrator during an initial time $\kappa_{i}^{-1}$.

\section{RESULTS}

Fig. 1 shows the responses of a same step input of similar reels with different cable length and position on the mechanism structure. The reel, coupled with a cable, has a response that varies depending on the applied tension, the cable length and the position where the reel is installed on the mechanism structure which justifies this optimization algorithm to reach the best performance for a haptic device. As such, a better model to be used would be a higher-order transfer function, but this is ignored, as the final optimization of the FPID parameters of the reel controller will be achieved using the ES-Tuning algorithm.

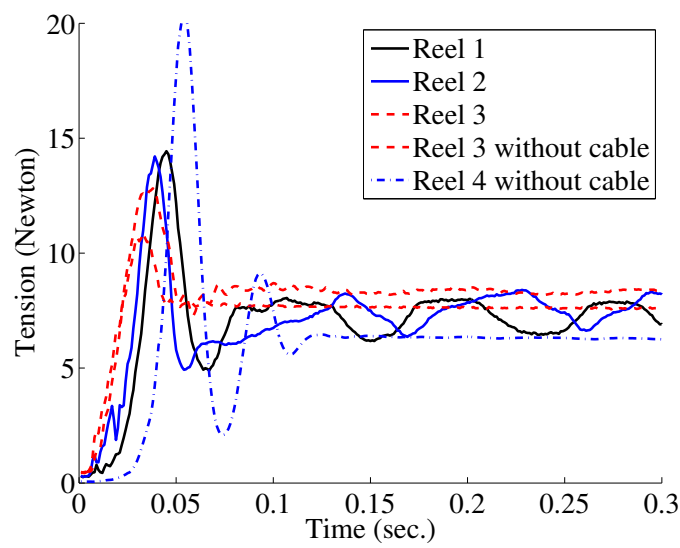

Fig. 1. Transient responses for four different reels

Fig. 2 presents the evolution of the cost function that ultimately converges toward a local minimum with different initial FPID parameters. Note that the final results depend on these initial parameters and the choice of the amplitude of $\alpha_{i}$ and $\gamma_{i}$. For this simulation, $\alpha_{i}$ and $\gamma_{i}$ are chosen quite small and the convergence time is high but the precision of the optimal parameters is adequate. The algorithm completes its execution when the linear regression of a set of $J$ values passes through a null slope and increases thereafter. Actually, this number of values uses for the linear regression depends on the convergence time.

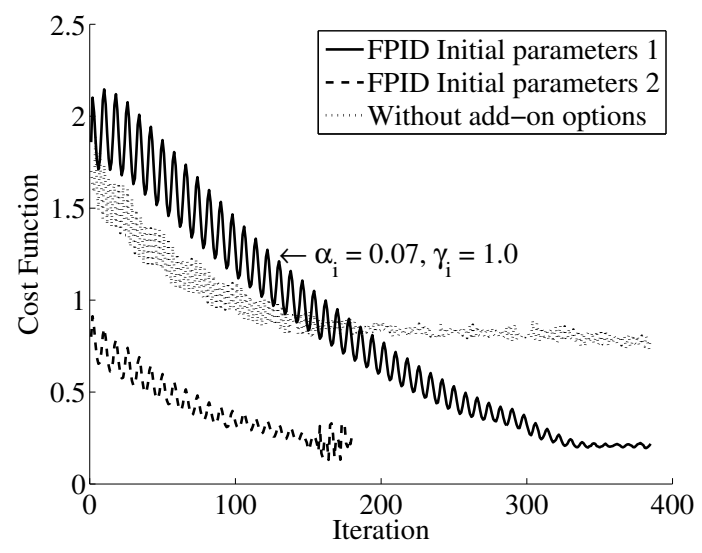

Fig. 2. Evolution of the cost function

From these results, when $\alpha_{i}$ and $\gamma_{i}$ have a high value, the integrator value for the reel increases to much, as described in Fig. 3, and then destabilizes the controller but the convergent time is short as shown in Fig. 4. Both values could be adjusted online with respect to the cost function. As the cost function decreases, $\alpha_{i}$ and $\gamma_{i}$ should also decrease for fine tuning each FPID parameters.

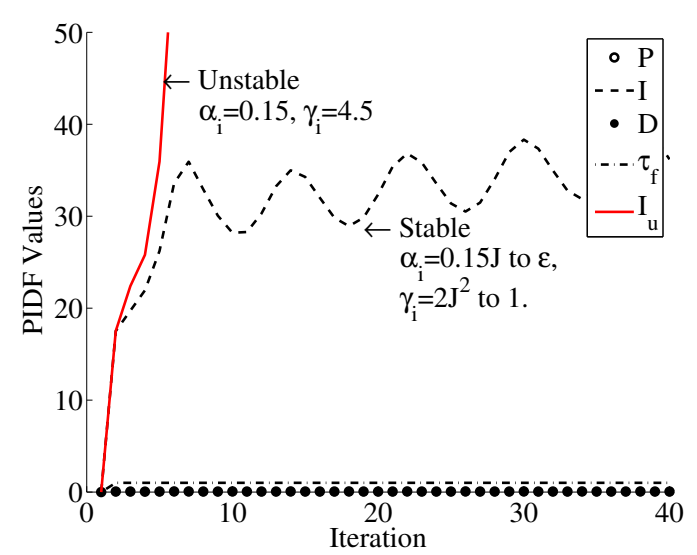

Fig. 3. PIDF parameters evolution without and with auto-tuning of $\alpha_{i}$ and $\gamma_{i}$

Fig. 5 shows the response of a tension sinusoid (with a frequency of $0.4 \mathrm{~Hz}$ ) that might occur when a user moves an haptic platform obtained with the optimal FPID parameters. The main objective is to increase the response time of this curve while reducing overshoot and increasing settling time of the step response. In this figure, at the fourth second, the tension error increases considerably. This behaviour can be explained in different ways: the mechanical deformation of the reel, the cable stiffness (axial and radial) and the friction of the cable on the moving parts of the reel. Such non-linearities decrease the performance of the controller. This result shows that the reel design should be revisited for haptic applications. 


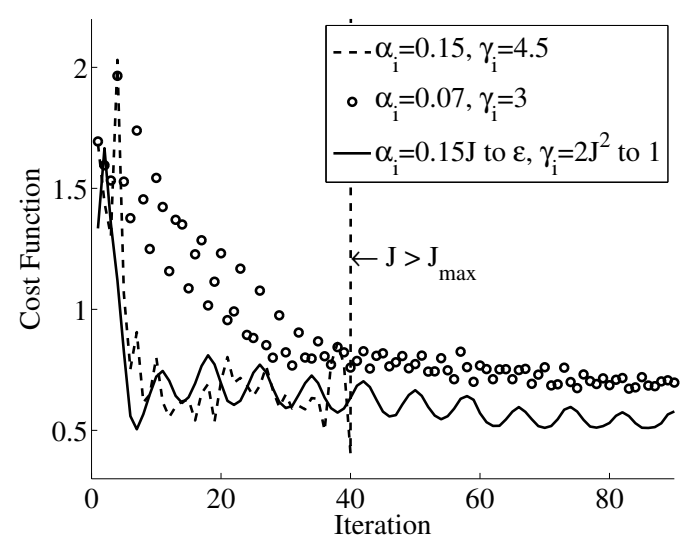

Fig. 4. Cost function without and with auto-tuning of $\alpha_{i}$ and $\gamma_{i}$

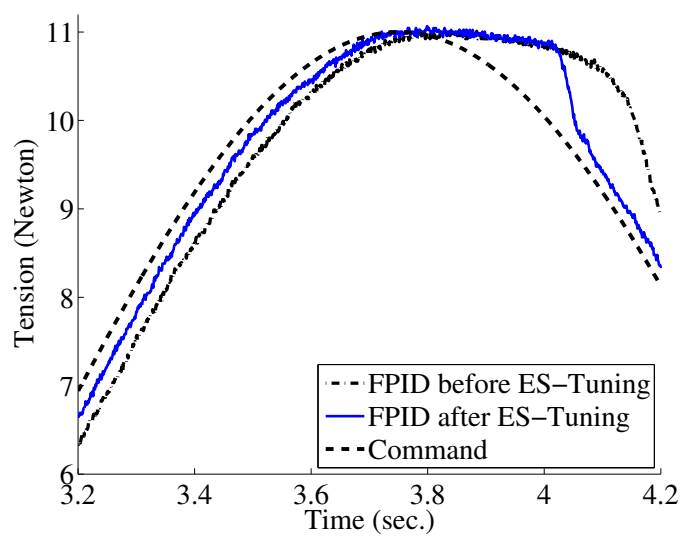

Fig. 5. Cable tension response for a sinusoid $(0.4 \mathrm{~Hz})$

\section{CONCLUSION}

The time convergence, noise robustness and transparency are critical factors that need to be considered when optimizing the control of a haptic mechanism with a method like ESTuning. A cost function is used as a parameter for defining dynamic transparency for an Heaviside setpoint. Also, some solutions are investigated for reducing the effect of noise on the computation of the cost function gradient. The enhanced ES-Tuning algorithm for haptic display includes:

- a cost function designed for describing the reel dynamic transparency;

- a setpoint filter added as a parameter that need to be optimized;

- a normalization factor $\eta$ multiplied the washout filter (high pass filter);

- a low pass filter applied before the demodulation in function of the actual cost function for adjusting the integration step and

- the logarithm of each parameter fed in the tuning algorithm for increasing robustness.

Another recommendation in implementing this approach is to apply a high Q low-pass filter with (20) on the cost function and to compute its mean before each iteration. The proposed algorithm executes five transient responses for which the mean of the cost function is computed before each optimization iteration.

\section{FUTURE WORK}

The method presented in this paper will be applied for a six degree of freedom redundant cable-driven parallel mechanism for which each articular actuator is a motorized reel. ES-Tuning will be implemented for optimal tuning of a Cartesian haptic impedance and admittance control scheme considering that each reel is optimally controlled in cable tension. Otis et al. [2008] have included such an hybrid impedance and admittance control scheme for operating a cable-driven locomotion interface.

\section{REFERENCES}

Syed Amjad Ali. Dynamic response of sagged cables. Computers \& Structures, 23(1):51 - 57, 1986.

C. F. Baicu, C. D. Rahn, and B. D. Nibali. Active boundary control of elastic cables: Theory and experiments. Journal of Sound and Vibration, 198(1):17 - 26, 1996.

J. Edward Colgate and J. Michael Brown. Factors affecting the $\mathrm{z}$-width of a haptic display. In Proceedings of the IEEE International Conference on Robotics and Automation, volume 4, pages 3205 - 3210, San Diego, CA, USA, 051994.

Shiqing Fang, D. Franitza, M. Torlo, F. Bekes, and M. Hiller. Motion control of a tendon-based parallel manipulator using optimal tension distribution. IEEE/ASME Transactions on Mechatronics, 9(3):561 - 8, 9 2004. ISSN 1083-4435.

Nick Killingsworth and Miroslav Krstic. Auto-tuning of pid controllers via extremum seeking. In Proceedings of the American Control Conference, volume 4, pages 2251 - 2256, Portland, OR, United States, 2005.

Nick J. Killingsworth, Salvador M. Aceves, Daniel L. Flowers, and Miroslav Krstic. Extremum seeking tuning of an experimental hcci engine combustion timing controller. In Proceedings of the American Control Conference, pages 3665 3670, New York, NY, United States, 2007.

Nick J. Killingsworth and Miroslav Krstic. Pid tuning using extremum seeking: Online, model free-performance optimization. IEEE Control Systems Magazine, 26(1):70 - 79, 2006. ISSN 0272-1708.

O. Lequin, M. Gevers, M. Mossberg, E. Bosmans, and L. Triest. Iterative feedback tuning of pid parameters: comparison with classical tuning rules. Control Engineering Practice, 11(9): 1023 - 33, 09 2003. ISSN 0967-0661.

So-Ryeok Oh and S.K. Agrawal. Cable suspended planar robots with redundant cables: controllers with positive tensions. IEEE Transactions on Robotics, 21(3):457 - 65, 062005. ISSN 1552-3098.

Martin JD Otis, Charles du Tremblay, Francois-M. De Rainville, M. Mokhtari, Denis Laurendeau, and Clement Gosselin. Hybrid control with multi-contact interactions for 6dof haptic foot platform on a cable-driven locomotion interface. In Proceedings of the IEEE Visualization and Graphics Technical Committee, Reno, Nevada, USA, 032008.

Jing-Chung Shen and Huann-Keng Chiang. Pid tuning rules for second order systems. In The 5th Asian Control Conference, volume 1, pages 472-477, Melbourne, Australia, 72004.

K. Vlachos and E. Papadopoulos. Transparency maximization methodology for haptic devices. IEEE/ASME Transactions on Mechatronics, 11(3):249 - 55, 6 2006. ISSN 1083-4435. 